\title{
ON THE DISTRIBUTIONS OF THE ZEROS OF SUMS OF EXPONENTIALS OF POLYNOMIALS*
}

\author{
BY \\ L. A. MACCOLL
}

1. INTRODUCTION

Certain work by other investigators suggests the problem of determining the distribution of the zeros of a function of the form

$$
f(z)=\sum_{j=0}^{J} \exp \left(\lambda_{j N} z^{N}+\cdots+\lambda_{j 1} z+\lambda_{j 0}\right),
$$

where $J$ and $N$ are positive integers, and the $\lambda$ 's are real or complex constants. The present paper gives the chief results of a study of this problem. In order to add precision to the problem, and in order to exclude certain extreme cases which are of minor interest, it is assumed (1) that we do not have $\lambda_{0 N}=\lambda_{1 N}=\cdots=\lambda_{J N} ;(2)$ that we do not have $\lambda_{0 n}=\lambda_{1 n}=\ldots=\lambda_{J_{n}} \neq 0$ for any $n<N$; (3) that for no two distinct values, $j^{\prime}$ and $j^{\prime \prime}$, of $j$ do we have all of the $N$ relations $\lambda_{j^{\prime \prime} n}=\lambda_{j^{\prime \prime} n}, n=1,2, \cdots, N$. For the sake of brevity, a function of the form (1) which satisfies these conditions will be called an $E$-function. The integer $N$ will be called the exponent of the $E$-function.

The problem discussed here is essentially a generalization, in one direction, of a problem that has already been the subject of numerous studies. C. E. Wilder, Tamarkin, Pólya, Schwengeler, and others $\nmid$ have studied the distributions of the zeros of functions of the form

$$
f(z)=\sum_{j=0}^{J} A_{j}(z) \exp \left(\lambda_{j} z\right),
$$

where $J$ is a positive integer, the $\lambda$ 's are constants, and the $A_{j}(z)$ 's are analytic functions which behave essentially as powers of $z$ when $|z|$ is large. Our generalization consists of replacing the linear exponents $\lambda_{j} z$ by the general polynomials $\lambda_{j N} z^{N}+\cdots+\lambda_{j 0}$. At the present time we shall not consider the still more general case in which we have non-constant coefficients $A_{j}(z)$ of the type described above; for the theory is complicated at best, and the chief

* Presented to the Society, October 29, 1932; received by the editors April 10, 1933, and, after revision, November 16, 1933.

$\dagger$ The previous work is conveniently summarized in the following expository paper: Langer, On the zeros of exponential sums and integrals, Bulletin of the American Mathematical Society, vol. 37 (1931), pp. 213-239. As this paper contains a rather full bibliography, it is unnecessary to give one here. 
of the new phenomena, due to the generalization of the exponents, appear even when the coefficients are constants. ${ }^{*}$ Broadly speaking, we find that the results for the general value of $N$ are similar to, but more complicated than, the known results for the special case in which $N=1$. The methods used here do not differ fundamentally from those that have been used in the earlier studies; but, naturally, the analysis is considerably more intricate, both formally and otherwise.

\section{NORMAL FORM OF $f(z)$}

We have written $f(z)$ in the form (1) in order to display the structure of the function in the clearest possible manner. In order to be able to work with the function effectively, however, we shall rewrite it in a certain normal form.

The numbers $\lambda_{0 N}, \cdots, \lambda_{J N}$ may be all distinct or not. In either case, by properly collecting terms if these $\lambda$ 's are not all distinct, we can write $f(z)$ in the form

$$
f(z)=\sum_{m=0}^{M} f_{m}(z) \exp \left[g_{m}(z)+\mu_{m} z^{N}\right],
$$

where $M$ is a positive integer, the numbers $\mu_{0}, \cdots, \mu_{M}$ are distinct, $g_{m}(z)$ is a polynomial which, if it is not zero, is of degree less than $N$, and $f_{m}(z)$ is either the constant 1 or an $E$-function having an exponent $N_{m}<N$.

If any one of the functions $f_{m}(z)$ is an $E$-function, we arrange it as we have just arranged $f(z)$. Thus we write

$$
f_{m}(z)=\sum_{n=0}^{M_{m}} f_{m n}(z) \exp \left[g_{m n}(z)+\mu_{m n} z^{N_{m}}\right],
$$

with stipulations similar to the above.

Likewise, if any one of the functions $f_{m n}(z)$ is an $E$-function, we write it in the form

$$
f_{m n}(z)=\sum_{p=0}^{M_{m n}} f_{m n p}(z) \exp \left[g_{m n p}(z)+\mu_{m n p} z^{N_{m n}}\right],
$$

with similar stipulations.

We continue this process of arranging $f(z)$ until it automatically terminates after a finite number of steps. It will be observed that the resulting normal form of $f(z)$ is not unique; for a given sum of exponentials of polynomials, which is not a constant and not the exponential of a polynomial, can be separated in various ways into two factors, one of which is an $E$-function and the other of which is the exponential of a polynomial. For example, if we have $f_{m}(z) \exp g_{m}(z)=\exp z^{2}+\exp \left(z^{2}+z\right)$, we can set

\footnotetext{
* Constant coefficients are effectively provided for by the constants $\lambda_{j 0}$.
} 


$$
f_{m}(z)=e^{-\lambda z}+e^{(1-\lambda) z}, \quad g_{m}(z)=z^{2}+\lambda z,
$$

where $\lambda$ is any constant. However, for our purposes the normal form of $f(z)$ is effectively unique.

\section{Critical polygons and critical Rays}

The distribution of the zeros of $f(z)$ is closely related to certain geometrical figures which will now be defined.

Let the points $\mu_{0}, \cdots, \mu_{M}$ be plotted in the complex plane. These points are distinct, and there are at least two of them. Let the smallest convex polygon that contains these points in its interior or on its boundary be drawn. If the points $\mu_{0}, \cdots, \mu_{M}$ are collinear, the polygon is to be regarded, in an obvious way, as having just two sides, which are coincident and face in opposite directions. We call this polygon the primary critical polygon for $f(z)$, and we denote it by the symbol $P$.

For the present, we assign subscripts so that $\mu_{0}, \cdots, \mu_{M}$, are the vertices of $P$ in counter-clockwise order, an arbitrarily chosen vertex being called $\mu_{0}$. If $M^{\prime}<M$, the points $\mu_{M^{\prime}+1}, \cdots, \mu_{M}$ are in the interior of $P$ or on the sides of $P$ between the vertices. Let the side of $P$ that follows the typical vertex $\mu_{\alpha}$ in counter-clockwise order be denoted by $L_{\alpha}$. From any point on $L_{\alpha}$ draw a normal exterior to $P$. Let $\phi_{\alpha}\left(0 \leqq \phi_{\alpha}<2 \pi\right)$ be the angle between the positive real axis and this outward-drawn normal. Let the vertex denoted by $\mu_{0}$ be selected so that the $\phi$ 's increase with their subscripts. From the origin we now draw the $\left(M^{\prime}+1\right) N$ rays $R_{\alpha}^{(\beta)}$ represented by the equations

$$
R_{\alpha}^{(\beta)}: \operatorname{amp} z=-\left(\phi_{\alpha}+2 \beta \pi\right) / N ; \alpha=0, \cdots, M^{\prime} ; \beta=0, \cdots, N-1 .
$$

We call these rays the primary critical rays for $f(z)$. It is to be noted that if the value of $\beta$ is fixed, the $M^{\prime}+1$ rays corresponding to the several values of $\alpha$, in increasing order, succeed one another in clockwise order and are contained in a sector of angular opening $2 \pi / N$, and that the $N$ sectors of this kind, corresponding to the several values of $\beta$ in increasing order, succeed one another in clockwise order without overlapping.

The primary critical rays divide the plane into $\left(M^{\prime}+1\right) N$ sectors, each of which has its vertex at the origin, is bounded by two of the rays, and has none of the rays in its interior. We denote the one of these sectors that is bounded on the clockwise side by the ray $R_{\alpha}^{(\beta)}$ by the symbol $S_{\alpha}^{(\beta)}$. Each of these sectors is understood to be an open point set.

As just above, let $\alpha$ be an arbitrarily chosen one of the integers $0, \cdots, M^{\prime}$. If the function $f_{\alpha}(z)$ is not a constant, we construct its primary 
critical polygon $P_{\alpha}$, its primary critical rays*

$$
R_{\alpha \beta}^{(\gamma)}: \beta=0, \cdots, M_{\alpha}^{\prime} ; \gamma=0, \cdots, N_{\alpha}-1,
$$

and the associated sectors $S_{\alpha \beta}^{(\gamma)}$.

Let $\alpha$ be one of the integers $0, \cdots, M^{\prime}$, and let $\beta$ be one of the integers $0, \cdots, M_{\alpha}^{\prime}$ (assuming that $f_{\alpha}(z)$ is not a constant). If the function $f_{\alpha \beta}(z)$ is not a constant, we construct its primary critical polygon $P_{\alpha \beta}$, its primary critical rays $R_{\alpha \beta \gamma}^{(\delta)}$, and the associated sectors $S_{\alpha \beta \gamma}^{(\delta)}$.

We continue this process of constructing polygons, rays and sectors until it automatically terminates, as it must, after a finite number of steps. It is to be noted that if $m>M^{\prime}$, we do not construct the figures for $f_{m}(z)$; and that if $0 \leqq \alpha \leqq M^{\prime}, n>M_{\alpha}^{\prime}$, we do not construct the figures for $f_{\alpha n}(z)$. A similar remark applies also to the further cases.

We have defined the primary critical rays for $f(z)$; now we proceed to define critical rays of "higher order" for $f(z)$. A secondary critical ray for $f(z)$ is a primary critical ray for a function $f_{\alpha}(z), \alpha=0, \cdots, M^{\prime}$, which lies in one of the sectors $S_{\alpha}^{(0)}, \cdots, S_{\alpha}^{(N-1)}$. A tertiary critical ray for $f(z)$ is a secondary critical ray for a function $f_{\alpha}(z), \alpha=0, \cdots, M^{\prime}$, which lies in one of the sectors $S_{\alpha}^{(0)}, \cdots, S_{\alpha}^{(N-1)}$. Critical rays of other orders are defined similarly. An essential feature of these definitions is the fact that we have the same subscript in the symbols $f_{\alpha}(z), S_{\alpha}{ }^{(0)}, \cdots, S_{\alpha}^{(N-1)}$. It is understood that the definitions of the critical rays of higher orders of the functions $f_{\alpha}(z)$ are precisely analogous to the definitions of the critical rays of higher order of $f(z)$; hence the definitions of the latter rays are complete. In this way we get a finite set of critical rays for $f(z)$, and these critical rays are classified as primary, secondary, tertiary, etc. At the very least there are two primary critical rays. Whether or not there are any critical rays of higher order depends on the structure of the particular function under consideration.

\section{ZERO-FREE REGIONS}

The first result concerning the distribution of the zeros of $f(z)$ is stated in the following:

THEOREM 1. There exists a set of half-strips $\dagger$, equal in number to the critical rays of $f(z)$, each extending in the direction of a different one of these critical rays, such that each zero of $f(z)$ is a point of one or more of these half-strips.

* $M_{\alpha}^{\prime}$ has the same significance in regard to $f_{\alpha}(z)$ that the previously defined symbol $M^{\prime}$ has in regard to $f(z)$.

† By a half-strip. we shall always mean the open set of points between two parallel straight lines and on one side of a line perpendicular to these. 
The theorem is an immediate consequence of the following

LEMMa. There exists a set of half-strips, equal in number to the critical rays of $f(z)$, each extending in the direction of a different one of these critical rays, and there exist two positive constants, $A$ and $B$, such that if $|z| \geqq A$, and if $z$ is not in any one of the half-strips, we have the relation

$$
|f(z)| \geqq \exp \left[-B|z|^{N}\right] \text {. }
$$

The lemma will be proved by a process of induction. The reasoning in the following $\$ 4.1$ proves the lemma directly for the case in which $N=1$. It will be shown that if $N>1$, the truth of the lemma is a consequence of the assumed truths of the corresponding lemmas concerning $E$-functions having exponents less than $N$.

The reasoning just referred to consists of two main steps. In the first place, we shall show that if we enclose each primary critical ray of $f(z)$ in a sector, with vertex at the origin and of arbitrarily small angular opening, and if we construct certain half-strips each extending in the direction of a different non-primary critical ray, we have a relation of the form (5), provided $|z|$ is large and provided $z$ is not in any one of these sectors or half-strips. In the second place, we shall show that a relation such as (5) obtains at each suffciently distant point of a small sector enclosing a primary critical ray, provided the point does not lie in a certain half-strip extending in the direction of the ray.

As most of the analysis used in the proof possesses no unusual features, many of the details will be left to the reader.

\subsection{Proof of THE LeMMA}

Let $\alpha$ be an arbitrarily chosen one of the integers $0, \cdots, M^{\prime}$, and let $\beta$ be an arbitrarily chosen one of the integers $0, \cdots, N-1$. Let $\epsilon$ be a positive number such that $2 \epsilon$ is less than the angular opening of the sector $S_{\alpha}^{(\beta)}$. Consider the sector $\Sigma_{\alpha}^{(\beta)}$ that is defined by the appropriate one of the following relations:

$$
\begin{aligned}
& \alpha=1, \cdots, M^{\prime} ; \beta=0, \cdots, N-1: \\
& \Sigma_{\alpha}^{(\beta)}:-\left(\phi_{\alpha}+2 \beta \pi-N \epsilon\right) / N \leqq \operatorname{amp} z \leqq-\left(\phi_{\alpha-1}+2 \beta \pi+N \epsilon\right) / N ; \\
& \alpha=0 ; \beta=1, \cdots, N-1: \\
& \Sigma_{\alpha}^{(\beta)}:-\left(\phi_{0}+2 \beta \pi-N \epsilon\right) / N \leqq \operatorname{amp} z \leqq-\left(\phi_{M^{\prime}}+2 \beta \pi-2 \pi+N \epsilon\right) / N ; \\
& \alpha=\beta=0: \\
& \Sigma_{\alpha}^{(\beta)}:-2 \pi+\epsilon-\phi_{0} / N \leqq \operatorname{amp} z \leqq-2 \pi-\epsilon-\left(\phi_{M^{\prime}}-2 \pi\right) / N .
\end{aligned}
$$

Each point of $\Sigma_{\alpha}^{(\beta)}$, except the origin, is a point of $S_{\alpha}^{(\beta)}$. 
If the sector $S_{\alpha}^{(\beta)}$ contains no critical ray of $f(z)$, by the hypothesis for the induction we have a relation of the following form, provided $z$ is in $\Sigma_{\alpha}^{(\beta)}$ and $|z|$ is sufficiently large:

$$
\left|f_{\alpha}(z) \exp g_{\alpha}(z)\right| \geqq \exp \left[-B|z|^{N-1}\right], B \text { a positive constant. }
$$

If $S_{\alpha}^{(\beta)}$ contains one or more critical rays of $f(z)$, we take $\epsilon$ so small that $\Sigma_{\alpha}^{(\beta)}$ contains all of these rays in its interior. Then to each of these rays there corresponds a half-strip, extending in the direction of the ray, such that if $z$ is in $\Sigma_{\alpha}^{(\beta)}$, but is not in any one of these half-strips, and if $|z|$ is sufficiently large, we have a relation of the form (7). We denote the set of points in $\Sigma_{\alpha}^{(\beta)}$, and not in any of these half-strips, by the symbol $T_{\alpha}^{(\beta)}$. If $S_{\alpha}^{(\beta)}$ contains no critical rays of $f(z)$, we use $T_{\alpha}^{(\beta)}$ as an alternative symbol for the sector $\Sigma_{\alpha}^{(\beta)}$.

We write $f(z)$ in the form

$$
\begin{aligned}
f(z)= & \left\{\exp \left(\mu_{\alpha} z^{N}\right)\right\}\left\{f_{\alpha}(z) \exp g_{\alpha}(z)\right. \\
& \left.+\sum_{m=0}^{M} f_{m}(z) \exp \left[g_{m}(z)+\left(\mu_{m}-\mu_{\alpha}\right) z^{N}\right]\right\},
\end{aligned}
$$

where the prime on the summation sign indicates that the term for $m=\alpha$ is to be omitted. It is easy to show, from the geometry of the polygon $P$, that in the sufficiently distant part of $\Sigma_{\alpha}^{(\beta)}$ we have

$$
\left|\sum_{m=0}^{M} f_{m}(z) \exp \left[g_{m}(z)+\left(\mu_{m}-\mu_{\alpha}\right) z^{N}\right]\right| \leqq \exp \left[-B|z|^{N}\right],
$$

where $B$ is a positive constant. It follows from the last relation, and from (7), that in the sufficiently distant part of $T_{\alpha}^{(\beta)}$ we have a relation of the form (5). This completes the first of the two main parts of the proof of the lemma.

In the second part of the proof it is convenient to employ a new assignment of the subscripts. Choose an arbitrary side of the polygon $P$, and denote it by the symbol $L$. We assign subscripts so that $\mu_{0}, \cdots, \mu_{M^{\prime \prime}}$ are the $\mu^{\prime}$ 's that lie on $L, \mu_{0}$ and $\mu_{M^{\prime \prime}}$ being the clockwise and counter-clockwise extremities of $L$, respectively.

We write the function $f(z)$ in the form

$$
f(z)=[h(z)+k(z)] \exp \left[\frac{1}{2}\left(\mu_{0}+\mu_{M^{\prime \prime}}\right) z^{N}\right],
$$

where*

* Of course, it may happen that $M^{\prime \prime}=M$. In this event the function $k(z)$ does not appear, and the next few steps in the proof are simply to be omitted. 
(9)

$$
\begin{aligned}
& h(z)=\sum_{m=0}^{M \prime \prime} f_{m}(z) \exp \left[g_{m}(z)+\left(\mu_{m}-\frac{\mu_{0}+\mu_{M^{\prime \prime}}}{2}\right) z^{N}\right], \\
& k(z)=\sum_{m=M^{\prime \prime}+1}^{M} f_{m}(z) \exp \left[g_{m}(z)+\left(\mu_{m}-\frac{\mu_{0}+\mu_{M^{\prime \prime}}}{2}\right) z^{N}\right] .
\end{aligned}
$$

From any point on $L$ draw a normal exterior to $P$, and let $\phi(0 \leqq \phi<2 \pi)$ denote the angle between the positive real axis and this outward-drawn normal. It is clear that if $m>M^{\prime \prime}$, and if amp $\left[\mu_{m}-\frac{1}{2}\left(\mu_{0}+\mu_{M^{\prime \prime}}\right)\right]$ is suitably defined,

$$
\phi+\frac{\pi}{2}+\sigma \leqq \operatorname{amp}\left[\mu_{m}-\frac{1}{2}\left(\mu_{0}+\mu_{M^{\prime \prime}}\right)\right] \leqq \phi+\frac{3 \pi}{2}-\sigma,
$$

where $\sigma$ is a certain number that satisfies the relations $0<\sigma \leqq \pi / 2$.

Let $\epsilon$ be a positive number less than $\sigma$. Consider any one of the sectors $U_{\beta}$ that are defined by the relations

$$
\begin{gathered}
U_{\beta}:(-\phi-\sigma-2 \beta \pi+\epsilon) / N \leqq \operatorname{amp} z \leqq(-\phi+\sigma-2 \beta \pi-\epsilon) / N, \\
\beta=0, \cdots, N-1 .
\end{gathered}
$$

It is easy to show that if $z$ is in $U_{\beta}$, and if $|z|$ is sufficiently large, we have a relation of the form

$$
|k(z)| \leqq \exp \left[-B|z|^{N}\right], B \text { a positive constant. }
$$

We now write the function $h(z)$ in the form

$$
\begin{aligned}
h(z)= & f_{0}(z) \exp \left[g_{0}(z)+\frac{1}{2}\left(\mu_{0}-\mu_{M^{\prime \prime}}\right) z^{N}\right] \\
& \cdot\left\{1+\sum_{m=1}^{M \prime \prime} \frac{f_{m}(z)}{f_{0}(z)} \exp \left[g_{m}(z)-g_{0}(z)+\left(\mu_{m}-\mu_{0}\right) z^{N}\right]\right\} ;
\end{aligned}
$$

and, in order to simplify the formal side of the exposition slightly, we assume, for the moment, that the primary critical ray

$$
\operatorname{amp} z=-(\phi+2 \beta \pi) / N
$$

is not a critical ray of the function $f_{0}(z)$.

By the hypothesis for the induction, if the angular opening of the sector $U_{\beta}$ is taken sufficiently small, if $z$ is in $U_{\beta}$, and if $|z|$ is sufficiently large, we have a relation of the form

$$
\left|f_{0}(z)\right| \geqq \exp \left[-B_{0}|z|^{N_{0}}\right], B_{0} \text { a positive constant. }
$$

There exist positive numbers, $C_{m}$ and $D_{m}$, such that, for all values of $z$,

$$
\left|f_{m}(z) \exp \left[g_{m}(z)-g_{0}(z)\right]\right| \leqq \exp \left[C_{m}|z|^{N-1}+D_{m}\right] \text {. }
$$


It follows, therefore, that if $z$ is in $U_{\beta}$, and $|z|$ is sufficiently large, we have the following relation, ${ }^{*}$ for $m=1, \cdots, M^{\prime \prime}$ :

$$
\begin{aligned}
& \left|\frac{f_{m}(z)}{f_{0}(z)} \exp \left[g_{m}(z)-g_{0}(z)+\left(\mu_{m}-\mu_{0}\right) z^{N}\right]\right| \\
& \quad \leqq \exp \left\{B_{0}|z|^{N_{0}}+C_{m}|z|^{N-1}+D_{m}+R\left[\left(\mu_{m}-\mu_{0}\right) z^{N}\right]\right\} .
\end{aligned}
$$

Let us write

$$
z=r e^{i \theta}, \quad \mu_{m}-\mu_{0}=R_{m} e^{i(\phi+\pi / 2)}, \quad m=1, \cdots, M^{\prime \prime},
$$

where $r$ is real and non-negative, and $R_{m}$ is positive. Let $q$ be a real number such that $M^{\prime \prime} e^{q}<1$. We now write the $M^{\prime \prime}$ relations

$$
R_{m} r^{N} \cos (N \theta+\phi+\pi / 2)+B_{0} r^{N_{0}}+C_{m} r^{N-1}+D_{m} \leqq q,
$$

and proceed to investigate the several regions defined by them.

The boundary of the region defined by the typical relation (10) is represented by the equation

$$
N \theta+\phi=\arcsin \left\{\frac{1}{R_{m}}\left[B_{0} r^{N_{0}-N}+C_{m} r^{-1}+\left(D_{m}-q\right) r^{-N}\right]\right\} .
$$

For our purposes it is convenient to write this last relation, for large values of $r$, in the form

$$
r[\theta-(-\phi-j \pi) / N]=c_{0}+\frac{c_{1}}{r}+\frac{c_{2}}{r^{2}}+\cdots,
$$

where $j$ is any integer, and the $c$ 's are constants. Equation (12) represents a curve which is asymptotic to a line parallel to the ray $\theta=-(\phi+j \pi) / N$, or to the ray itself.

Giving $j$ successively the values $0, \cdots, 2 N-1$, we get, from (12), the representations of the $2 N$ branches of the curve represented by (11). The branch corresponding to the even value $2 \beta$ of $j$ is asymptotic to a parallel to the bisector of $U_{\beta}$, or to the bisector itself. We are not directly concerned with the branches that correspond to odd values of $j$.

In the sufficiently distant part of the sector $U_{\beta}$ there are $M^{\prime \prime}$ branches of curves of the type just described, corresponding to the several values $1, \cdots, M^{\prime \prime}$ of $m$.

As $R_{1}, \cdots, R_{M^{\prime \prime}}$ are all positive, the region (10) lies on the counterclockwise sides of the branches of the boundary that correspond to even values of $j$. The region lies on the clockwise sides of the branches that correspond to odd values of $j$.

\footnotetext{
* If $u$ and $v$ are real, and $w=u+i v$, we write $u=R w, v=J w$.
} 
It follows from the above that we can draw a line parallel to the bisector of $U_{\beta}$, such that in the distant part of the sub-sector bounded by this line and the counter-clockwise side of $U_{\beta}$ the function

$$
1+\sum_{m=1}^{M^{\prime \prime}} \frac{f_{m}(z)}{f_{0}(z)} \exp \left[g_{m}(z)-g_{0}(z)+\left(\mu_{m}-\mu_{0}\right) z^{N}\right]
$$

is bounded away from zero.*

Now consider the function

$$
f_{0}(z) \exp \left[g_{0}(z)+\frac{1}{2}\left(\mu_{0}-\mu_{M^{\prime \prime}}\right) z^{N}\right] .
$$

If $z$ is in $U_{\beta}$, and if $|z|$ is sufficiently large, the modulus of this function is not less than

$$
\exp \left\{-B_{0}^{\prime} r^{N-1}+R\left[\frac{1}{2}\left(\mu_{0}-\mu_{M^{\prime \prime}}\right) z^{N}\right]\right\},
$$

where $B_{0}^{\prime}$ is a suitably chosen positive constant. By reasoning similar to that used just above, we show that in $U_{\beta}$ there is a sub-sector, ${ }^{*}$ bounded by a line parallel to the bisector of $U_{\beta}$ and by the counter-clockwise side of $U_{\beta}$, in the distant part of which the function (13) is bounded away from zero.

It follows that in $U_{\beta}$ there is a sub-sector, ${ }^{*}$ bounded by a line parallel to the bisector of $U_{\beta}$ and by the counter-clockwise side of $U_{\beta}$, in the distant part of which the function $h(z)$ is bounded away from zero.

Assuming provisionally that the ray amp $z=-(\phi+2 \beta \pi) / N$ is not a critical ray of $f_{M^{\prime \prime}}(z)$, we show, in an entirely similar way, that in $U_{\beta}$ there is a sub-sector, ${ }^{*}$ bounded by a parallel to the bisector of $U_{\beta}$ and by the clockwise side of $U_{\beta}$, in the distant part of which $h(z)$ is bounded away from zero.

It is easy to show that we have the results just stated even when the ray amp $z=-(\phi+2 \beta \pi) / N$ is a critical ray of one or both of the functions $f_{0}(z)$, $f_{M \prime \prime}(z)$. By the hypothesis for the induction, the sector then contains a halfstrip, extending in the direction of the bisector of $U_{\beta}$, such that in the distant parts of the regions within the sector and outside the half-strip, we have relations of the form

$$
\left|f_{0}(z)\right| \geqq \exp \left[-B|z|^{N_{0}}\right], \quad\left|f_{M^{\prime \prime}}(z)\right| \geqq \exp \left[-B|z|^{N_{M^{\prime \prime}}}\right],
$$

where $B$ is a positive constant. Now we have only to confine our attention to values of $z$ which lie in one or the other of the regions just described. With this understanding about the values of $z$ under discussion, the preceding reasoning is valid without any essential change, and we are led again to the result stated in the preceding paragraph.

It follows from (8), and what has been proved concerning the functions

* The sub-sector is taken as including the points on its boundary. 
$h(z)$ and $k(z)$, that in $U_{\beta}$ there are two sub-sectors, ${ }^{*}$ each bounded by a line parallel to the bisector of $U_{\beta}$ and by a different one of the sides of the sector, such that in the sufficiently distant parts of these sub-sectors we have a relation of the form (5).

This completes the proof of the lemma.

\section{Distribution OF the zeros Within A CRITICAL halF-STRIP}

We shall call the half-strips determined in the preceding paragraphs, within which the zeros of $f(z)$ must lie, critical half-strips. It is natural to classify the several critical half-strips as primary, secondary, tertiary, etc., according to the classification of the several critical rays to which they correspond.

It has not yet been shown that $f(z)$ has any zeros at all. We shall now prove that zeros do actually exist, and we shall obtain asymptotic formulas giving the distributions of the zeros in the various critical half-strips. Specifcally, the remainder of the paper will be devoted to proving the following two theorems.

THEOREM 2. The number of zeros of $f(z)$ in the interior of a rectangle bounded by segments of length $r$ of the sides of a primary critical half-strip, by the end of the half-strip, and by a segment congruent to the end, is equal, for $r$ large, to

$$
\left(l r^{N} /(2 \pi)\right)[1+O(1 / r)],
$$

where $l$ is the length of the side of the primary critical polygon that corresponds $\dagger$ to the half-strip.

TheORem 3. Let $Z_{f}(r)$ and $Z_{f_{\alpha} \cdots \lambda_{\mu}}(r)$ denote, respectively, the numbers of zeros of $f(z)$ and $f_{\alpha} \ldots \lambda_{\mu}(z)$ in the interior of a rectangle bounded (1) by segments of length $r$ of the sides of a critical half-strip which is primary for $f_{\alpha} \ldots \lambda_{\mu}(z)$ and non-primary for $f(z), f_{\alpha}(z), \cdots, f_{\alpha} \ldots \lambda(z) \ddagger ;(2)$ by the end of the half-strip; (3) by a segment congruent to the end. Then, if $r$ is large, we have

$$
Z_{f}(r)=Z_{f_{\alpha \ldots \lambda \mu}}(r)[1+O(1 / r)] .
$$

* See footnote on p. 349.

$\dagger$ Each side of the polygon determines, through the direction of the outward-drawn normal, a set of primary critical rays, and each primary critical half-strip extends in the direction of one of these rays. This is the correspondence referred to.

$\ddagger$ It is to be observed that a non-primary critical ray for $f(z)$ is a critical ray for a definite function $f_{\alpha}(z)$; if it is a non-primary critical ray for $f_{\alpha}(z)$, it is a critical ray for a definite function $f_{\alpha \beta}(z)$; and so on. The ray is a primary critical ray for a definite function $f_{\alpha} \cdots \lambda_{\mu}(z)$. Also, it is to be observed that the critical half-strip for $f(z)$, corresponding to the ray, can be considered as the critical halfstrip for each of the functions $f_{\alpha}(z), f_{\alpha \beta}(z), \cdots, f_{\alpha} \cdots \lambda_{\mu}(z)$, corresponding to the ray. 
We shall prove these theorems by a process of induction. Our reasoning proves Theorem 2 directly for the case in which $N=1$. We shall show that if $N>1$, the theorems are consequences of the corresponding theorems concerning $E$-functions having exponents less than $N$. $^{*}$

\subsection{Proof of Theorem 2}

We here employ the assignment of subscripts and the notation that were used in the latter part of $\$ 4.1$.

Consider a particular primary critical half-strip, say the one that extends in the direction of the bisector of the sector $U_{\beta}$.

Consider a rectangle, $R$, the vertices of which, in counter-clockwise order, are denoted by $V_{1}, V_{2}, V_{3}, V_{4}$, respectively. The rectangle is taken so that (1) $V_{1} V_{2}$ is a segment of the clockwise side of the half-strip; (2) $V_{3} V_{4}$ is a segment of the counter-clockwise side of the half-strip; (3) $f(z)$ does not vanish on either of the segments $V_{2} V_{3}, V_{4} V_{1}$. We take the half-strips so that $f(z)$ does not vanish on either side of any one of them; hence $f(z)$ does not vanish on the boundary of $R$. Let the complex number corresponding to the typical vertex $V_{i}$ of $R$ be $z_{i}$.

The number, $Z_{f}(R)$, of zeros of $f(z)$ contained in $R$ is given by the formula $2 \pi Z_{f}(R)=$ variation of $\operatorname{amp} f(z)$ along the curve $V_{1} V_{2} V_{3} V_{4} V_{1}$.

For the sake of brevity, we use a self-explanatory notation in which this formula becomes

$$
\begin{aligned}
2 \pi Z_{f}(R)= & {\left[\text { v.a. } f(z), V_{1} V_{2} V_{3} V_{4} V_{1}\right] } \\
= & {\left[\text { v.a. } f(z), V_{1} V_{2}\right]+\left[\text { v.a. } f(z), V_{2} V_{3}\right] } \\
& +\left[\text { v.a. } f(z), V_{3} V_{4}\right]+\left[\text { v.a. } f(z), V_{4} V_{1}\right] .
\end{aligned}
$$

We shall estimate the value of each of the terms in the right-hand member of (14), assuming that $R$ lies in the distant part of the half-strip.

First consider the term [v.a. $f(z), V_{1} V_{2}$ ]. We write $f(z)$ in the form

$$
\begin{aligned}
f(z)= & \left\{f_{M^{\prime \prime}}(z)\right\}\left\{\exp \left[g_{M^{\prime \prime}}(z)+\mu_{M^{\prime}} z^{N}\right]\right\}\left\{1+\sum_{m=0}^{M^{\prime \prime}-1} \frac{f_{m}(z)}{f_{M^{\prime \prime}}(z)} \exp \left[g_{m}(z)\right.\right. \\
& \left.\left.-g_{M^{\prime \prime}}(z)+\left(\mu_{m}-\mu_{M^{\prime \prime}}\right) z^{N}\right]\right\}\left\{1+\frac{k(z)}{h(z)}\right\} \equiv F_{1} F_{2} F_{3} F_{4} .
\end{aligned}
$$

The functions $h(z), k(z)$ are defined by equations (9). We now have the following relation:

* The widths of the half-strips, and the locations of the ends of the half-strips are somewhat arbitrary. The formulas stated in the theorems imply, of course, that the half-strips have been definitely chosen. 


$$
\text { [v.a. } \left.f(z), V_{1} V_{2}\right]=\sum_{i=1}^{4}\left[\text { v.a. } F_{i}, V_{1} V_{2}\right] \text {. }
$$

It has been shown in $\$ 4.1$ that $\left|F_{3}-1\right|<1$, and that $\left|F_{4}-1\right|<1$, when $z$ is on the distant part of the clockwise side of the half-strip. Therefore, if $R$ is sufficiently distant,

$$
\begin{aligned}
& -\pi<\left[\text { v.a. } F_{3}, V_{1} V_{2}\right]<\pi, \\
& -\pi<\left[\text { v.a. } F_{4}, V_{1} V_{2}\right]<\pi .
\end{aligned}
$$

We have immediately

$$
\text { [v.a. } \left.F_{2}, V_{1} V_{2}\right]=\Im\left[g_{M^{\prime \prime}}\left(z_{2}\right)+\mu_{M^{\prime \prime}} z_{2}^{N}\right]-\Im\left[g_{M^{\prime \prime}}\left(z_{1}\right)+\mu_{M^{\prime}} z_{1}{ }^{N}\right] \text {. }
$$

It remains to consider the term [v.a. $f_{M^{\prime \prime}}(z), V_{1} V_{2}$ ]. If $f_{M^{\prime \prime}}(z)$ is constant, this term is zero. Henceforth assume that the function is non-constant. The function is of the form*

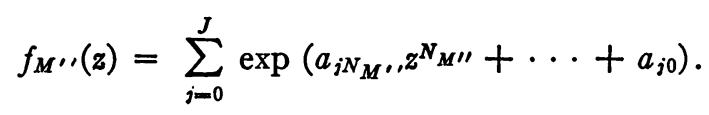

Let $z^{\prime}$ be the point collinear with $z_{1}$ and $z_{2}$ that is nearest the origin; let $z^{\prime \prime}$ be a particular one of the two points that are collinear with $z_{1}$ and $z_{2}$ and are such that $\left|z^{\prime}-z^{\prime \prime}\right|=1$. We write

$$
z=z^{\prime}+\left(z^{\prime \prime}-z^{\prime}\right) t
$$

thereby changing the independent variable from $z$ to $t$. We write

$$
f_{M \prime \prime}(z) \equiv \phi(t)=\sum_{j=0}^{J} \exp \left(b_{j N_{M},} t^{N_{M}^{\prime \prime}}+\cdots+b_{j 0}\right),
$$

where the $b^{\prime}$ 's are functions of $z^{\prime}, z^{\prime \prime}$, and the $a^{\prime}$ 's of (15). Let $b_{j k}=b_{j k}{ }^{\prime}+i b_{j k}{ }^{\prime \prime}$, where $b_{j k}{ }^{\prime}$ and $b_{j k}{ }^{\prime \prime}$ are real.

We now consider the functions

$$
\begin{aligned}
& A(t)=\sum_{j=0}^{J} \exp \left(b_{j N_{M^{\prime \prime}}^{\prime \prime}}^{\prime} t^{N_{M^{\prime \prime}} \prime}+\cdots+b_{j 0}^{\prime}\right) \cos \left(b_{j N_{M^{\prime \prime}}^{\prime \prime}}^{\prime \prime} t^{N_{M^{\prime \prime}}}+\cdots+b_{j 0}^{\prime \prime}\right), \\
& B(t)=\sum_{j=0}^{J} \exp \left(b_{j N_{M^{\prime}}^{\prime \prime}}^{\prime} t^{N_{M^{\prime \prime}} \prime}+\cdots+b_{j 0}^{\prime}\right) \sin \left(b_{j N_{M^{\prime \prime}}^{\prime \prime}}^{\prime \prime} t^{N_{M^{\prime \prime}}}+\cdots+b_{j 0}^{\prime \prime}\right) .
\end{aligned}
$$

When the points $z, z_{1}, z_{2}$ are collinear, $A(t)$ and $i B(t)$ are, respectively, the real and imaginary parts of $\phi(t) \equiv f_{M^{\prime \prime}}(z)$. Consequently, for such $z$ 's we have the relation

* It is not implied that the $J$ here is the same as the $J$ in equation (1). The same remark applies in several other places in the following work. 


$$
\tan \operatorname{amp} f_{M^{\prime \prime}}(z)=\tan \operatorname{amp} \phi(t)=\frac{B(t)}{A(t)} .
$$

Now $f_{M^{\prime \prime}}(z) \neq 0$ on the distant part of the clockwise side of the half-strip. Hence, $A(t)$ and $B(t)$ cannot vanish simultaneously on the part of the real axis in the $t$-plane that corresponds to the distant part of this side of the halfstrip. If either $A(t)$ or $B(t)$ is identically zero, amp $f_{M^{\prime \prime}}(z)$ is constant on the segment $V_{1} V_{2}$. Henceforth let us assume that neither $A(t)$ nor $B(t)$ is identically zero. It follows from (16), and the properties of the tangent function, that amp $f_{M^{\prime \prime}}(z)$ cannot vary by as much as $\pi$ on any distant segment of the side of the half-strip without $A(t)$ vanishing on the corresponding segment of the real axis in the $t$-plane. Therefore, if we can show that $A(t)$ has not more than, say, $\nu$ zeros on the segment in the $t$-plane that corresponds to the segment $V_{1} V_{2}$, it will follow that

$$
-(\nu+1) \pi<\left[\text { v.a. } f_{M^{\prime \prime}}(z), V_{1} V_{2}\right]<(\nu+1) \pi .
$$

Thus the problem of estimating the value of [v.a. $f_{M^{\prime \prime}}(z), V_{1} V_{2}$ ] is related to the problem of estimating the number of zeros of $A(t)$ on a certain distant segment on the real axis in the $t$-plane.

If $A(t)$ is the exponential of a polynomial, it has no zeros. Henceforth assume that $A(t)$ is not the exponential of a polynomial. Then $A(t)$ is a function of the same type as the function $f(z)$ which is the subject of this entire study, except for the essential difference that, instead of the exponent $N$, we have here the smaller exponent $N_{M^{\prime \prime}}$. Therefore, the zeros of $A(t)$, if there are any, are confined to certain half-strips in the $t$-plane. We are interested in those zeros, if there are any, which lie on a certain segment on the distant part of a particular half of the real axis. If no one of the critical half-strips for $A(t)$ contains this half of the real axis, there are no zeros on the segment in question (provided the rectangle $R$ is taken sufficiently distant in the $z$-plane). If, on the other hand, the above-mentioned half of the real axis in the $t$-plane is contained in some critical half-strip for $A(t)$, we draw a rectangle, $R^{\prime}$, in the latter half-strip (the rectangle having two of its sides on the sides of the half-strip, containing in its interior the segment corresponding to $V_{1} V_{2}$, and being taken so that $A(t)$ does not vanish on the boundary of $R^{\prime}$ ), and we estimate the number of zeros of $A(t)$ in $R^{\prime}$ by means of the theorems concerning $A(t)$ corresponding to our Theorems 2 and 3 . The number of zeros on the segment corresponding to $V_{1} V_{2}$ does not exceed the number of zeros in $R^{\prime}$. By the hypothesis for the induction, we have the following expression for the number of zeros of $A(t)$ contained in the rectangle $R^{\prime}$ : 


$$
\frac{a\left|t_{2}+\tau^{\prime \prime}\right|^{n}}{2 \pi}\left[1+O\left(1 /\left|t_{2}+\tau^{\prime \prime}\right|\right)\right]-\frac{a\left|t_{1}-\tau^{\prime}\right|^{n}}{2 \pi}\left[1+O\left(1 /\left|t_{1}-\tau^{\prime}\right|\right)\right]
$$

where $a$ is a positive constant, $n$ is a positive integer not greater than $N_{M^{\prime \prime}}, t_{1}$ and $t_{2}$ are the values of $t$ that correspond to $z_{1}$ and $z_{2}$, respectively, and $t_{2}+\tau^{\prime \prime}$ and $t_{1}-\tau^{\prime}$ are the values of $t$ corresponding to the points at which the real $t$-axis intersects the boundary of $R^{\prime} ;\left|\tau^{\prime}\right|$ and $\left|\tau^{\prime \prime}\right|$ may be taken arbitrarily small, but not zero.

We have now completed the estimation of the value of the term [v.a. $f(z), V_{1} V_{2}$ ]. It is evident that the term [v.a. $f(z), V_{3} V_{4}$ ] can be discussed in an altogether similar manner. No details of this discussion need be given here.

Next we shall estimate the value of the term

$$
\begin{aligned}
\text { [v.a. } \left.f(z), V_{2} V_{3}\right]= & \Im\left[\frac{1}{2}\left(\mu_{0}+\mu_{M^{\prime \prime}}\right) z_{3}{ }^{N}\right]-\Im\left[\frac{1}{2}\left(\mu_{0}+\mu_{M M^{\prime \prime}}\right) z_{2}{ }^{N}\right] \\
& +\left[\text { v.a. }(h(z)+k(z)), V_{2} V_{3}\right] .
\end{aligned}
$$

Let $z_{0}$ be the point at which the bisector of the sector $U_{\beta}$ intersects the straight line through $V_{2}$ and $V_{3}$. We write

$$
z_{3}-z_{2}=\zeta, \quad z=z_{0}+\zeta t,
$$

thus changing the independent variable from $z$ to $t$. We also write

$$
h(z) \equiv \psi(t), \quad k(z) \equiv \omega(t) .
$$

The function $\psi(t)+\omega(t)$ is of the form

$$
\psi(t)+\omega(t)=\sum_{j=0}^{J} \exp \left(\gamma_{j N} t^{N}+\cdots+\gamma_{j 0}\right),
$$

where the $\gamma$ 's are constants which depend on $z_{0}, \zeta$, and the $\lambda$ 's in (1). Let $\gamma_{j n}=\gamma_{j n}{ }^{\prime}+i \gamma_{j n}{ }^{\prime \prime}$, where $\boldsymbol{\gamma}_{j n}{ }^{\prime}$ and $\boldsymbol{\gamma}_{j n}{ }^{\prime \prime}$ are real.

We now consider the functions

$$
\begin{aligned}
& G(t)=\sum_{j=0}^{J} \exp \left(\gamma_{j N}^{\prime} t^{N}+\cdots+\gamma_{j 0}^{\prime}\right) \cos \left(\gamma_{j N}^{\prime \prime} t^{N}+\cdots+\gamma_{j 0}^{\prime \prime}\right), \\
& B(t)=\sum_{j=0}^{J} \exp \left(\gamma_{j N}^{\prime} t^{N}+\cdots+\gamma_{j 0}^{\prime}\right) \sin \left(\gamma_{j N}^{\prime \prime} t^{N}+\cdots+\gamma_{j 0}^{\prime \prime}\right) .
\end{aligned}
$$

If $z, z_{2}$, and $z_{3}$ are collinear, $G(t)$ and $i H(t)$ are, respectively, the real and imaginary parts of the function $\psi(t)+\omega(t)$.

For $0 \leqq m \leqq M^{\prime \prime}$, we have

$$
\mu_{m}-\frac{1}{2}\left(\mu_{0}+\mu_{M}^{\prime \prime}\right)=\rho_{m} \exp [i(\phi+\pi / 2)]
$$


where $\rho_{m}$ is real. Also, we have

$$
z_{0}=\left|z_{0}\right| \exp [-i(\phi+2 \beta \pi) / N] \text {. }
$$

Hence

$$
\left(\mu_{m}-\frac{\mu_{0}+\mu_{M^{\prime \prime}}}{2}\right) z^{N}=i \rho_{m}\left|z_{0}\right|^{N}+\cdots+\rho_{m} \zeta^{N} t^{N} \exp [i(\phi+\pi / 2)] .
$$

Let $T$ be any positive constant. The relation (17) shows that when $|t| \leqq T$, and when $\left|z_{0}\right|$ is sufficiently large, we have a relation of the form

$$
|\psi(t)| \leqq \exp \left[B_{1}\left|z_{0}\right|^{N-1}\right],
$$

where $B_{1}$ is a positive constant. We know that in the distant part of $U_{\beta}$ we have

$$
|k(z)| \leqq \exp \left[-B_{2}|z|^{N}\right],
$$

where $B_{2}$ is a positive constant; hence, when $|t| \leqq T$, and when $\left|z_{0}\right|$ is suffciently large, we have

$$
|\omega(t)| \leqq \exp \left[-B_{3}\left|z_{0}\right|^{N}\right],
$$

where $B_{3}$ is a positive constant. It follows that when $|t| \leqq T$, and when $\left|z_{0}\right|$ is sufficiently large, we have relations of the form

$$
|G(t)| \leqq \exp \left[B\left|z_{0}\right|^{N-1}\right], \quad|H(t)| \leqq \exp \left[B\left|z_{0}\right|^{N-1}\right],
$$

where $B$ is a positive constant.

When $z$ is collinear with $z_{2}$ and $z_{3}$ we have the relation

$$
\tan \operatorname{amp}[h(z)+k(z)]=\frac{H(t)}{G(t)} .
$$

Because of the way in which the rectangle $R$ has been taken, $G(t)$ and $H(t)$ do not vanish simultaneously at any point on the segment corresponding to $V_{2} V_{3}$. If either of the functions $G(t), H(t)$ is identically zero, amp $[h(z)+k(z)]$ is constant along $V_{2} V_{3}$. Henceforth assume that neither $G(t)$ nor $H(t)$ is identically zero. Then, if $G(t)$ vanishes not more than, say, $\nu$ times on the segment in the $t$-plane that corresponds to $V_{2} V_{3}$, the following relation holds:

$$
\left.-(\nu+1) \pi<\text { [v.a. }(h(z)+k(z)), V_{2} V_{3}\right]<(\nu+1) \pi \text {. }
$$

We have the same relation if $H(t)$ does not vanish more than $\nu$ times on the segment corresponding to $V_{2} V_{3}$.

We know that $h(z)$ is bounded away from zero when $z$ is on the distant part of the clockwise side of the half-strip. The same is, therefore, true of the function $h(z)+k(z)$. Hence, if $\left|z_{0}\right|$ is sufficiently large, and if $t_{2}$ is the value of 
$t$ that corresponds to the value $z_{2}$ of $z$, one at least of the numbers $\left|G\left(t_{2}\right)\right|$, $\left|H\left(t_{2}\right)\right|$ is greater than a certain fixed positive number $\delta$. To fix the ideas, suppose that $\left|G\left(t_{2}\right)\right|>\delta$; similar reasoning applies if $\left|G\left(t_{2}\right)\right| \leqq \delta$ and $\left|H\left(t_{2}\right)\right|>\delta$.

We wish to establish an upper bound for the number of zeros of $G(t)$.on the segment corresponding to $V_{2} V_{3}$. For this purpose we employ a result due to Jensen, which we state for our purposes in the following restricted form*:

Let $G(t)$ be an integral function, such that

$$
|G(t)| \leqq M(\tau) \text { for }\left|t-t_{2}\right| \leqq \tau .
$$

Let $G(t)$ vanish at the points $t^{(1)}, t^{(2)}, \cdots, t^{(v)}$, such that

$$
0 \leqq\left|t^{(n)}-t_{2}\right|<\tau \quad(n=1,2, \cdots, \nu) .
$$

Then

$$
\left|\left(t^{(1)}-t_{2}\right)\left(t^{(2)}-t_{2}\right) \cdots\left(t^{(v)}-t_{2}\right)\right| \geqq\left|G\left(t_{2}\right)\right| \tau^{v} / M(\tau) .
$$

Choose a positive number $\tau$ large enough so that the segment in the $t$-plane corresponding to $V_{2} V_{3}$ is contained in the interior of the circle $\left|t-t_{2}\right|=\tau$. The value of $\tau$ may be, and is, taken to be independent of $\left|z_{0}\right|$. Denote by $t_{3}$ the value of $t$ corresponding to $z_{3}$. We are interested in those zeros of $G(t)$, if there are any, that are within or on the circle $\left|t-t_{2}\right|=\left|t_{3}-t_{2}\right|$. We take the $t^{(n)}$ 's of the above theorem to be just these zeros. We then have, by (18) and (19),

$$
\left|t_{3}-t_{2}\right| \cdot \geqq \frac{\left|G\left(t_{2}\right)\right| \tau^{\prime}}{M(\tau)}>\frac{\delta \tau^{\prime}}{\exp \left[B\left|z_{0}\right|^{N-1}\right]},
$$

or

$$
\nu<\frac{B\left|z_{0}\right|^{N-1}-\log \delta}{\log \frac{\tau}{\left|t_{3}-t_{2}\right|}}
$$

the logarithms being real. This completes our estimation of the value of term [v.a. $f(z), V_{2} V_{3}$ ]. It is to be noted that $\tau$ is a constant greater than $\left|t_{3}-t_{2}\right|$, so the only variable in the right-hand member of (20) is $\left|z_{0}\right|$.

The term [v.a. $f(z), V_{4} V_{1}$ ] can be discussed in a way that is entirely similar to the way in which we have discussed [v.a. $f(z), V_{2} V_{3}$ ]. No details of this discussion need be given here.

To complete the proof of Theorem 2 we write out the complete expression for the number of zeros contained in the rectangle $R$, using the estimates we

* Bieberbach, Lehrbuch der Funktionentheorie, vol. II, p. 109. 
have found for the several terms in (14). We shall consider the side $V_{4} V_{1}$ of $R$ as fixed, and the side $V_{2} V_{3}$ as variable; in particular we shall confine our attention to cases in which the sides $V_{1} V_{2}$ and $V_{3} V_{4}$ are long. Collecting our results, we see that the number of zeros in $R$ is given by the equation

$$
\begin{aligned}
& 2 \pi Z_{f}(R)=\Im\left[\frac{1}{2}\left(\mu_{M^{\prime \prime}}-\mu_{0}\right)\left(z_{3}{ }^{N}+z_{2}{ }^{N}\right)\right]+\Im\left[g_{M^{\prime \prime}}\left(z_{2}\right)-g_{0}\left(z_{3}\right)\right] \\
& \quad+\left[\text { v.a. } f_{M^{\prime \prime}}(z), V_{1} V_{2}\right]+\left[\text { v.a. } f_{0}(z), V_{3} V_{4}\right]+\left[\text { v.a. }(h(z)+k(z)), V_{2} V_{3}\right]+W,
\end{aligned}
$$

where $W$ is a quantity that is less, in absolute value, than a fixed number.

Now, in terms of the notation used before,

$$
\begin{aligned}
\mu_{M^{\prime \prime}}-\mu_{0} & =\left|\mu_{M^{\prime \prime}}-\mu_{0}\right| \exp [i(\phi+\pi / 2)], \\
z_{2}{ }^{N} & =\left(z_{0}+\zeta t_{2}\right)^{N}=\left|z_{0}\right|^{N} \exp (-i \phi)+\cdots, \\
z_{3}{ }^{N} & =\left(z_{0}+\zeta t_{3}\right)^{N}=\left|z_{0}\right|^{N} \exp (-i \phi)+\cdots,
\end{aligned}
$$

and hence

$J\left[\frac{1}{2}\left(\mu_{M \prime \prime}-\mu_{0}\right)\left(z_{3}{ }^{N}+z_{2}{ }^{N}\right)\right]=\left|\mu_{M \prime \prime}-\mu_{0}\right| \cdot\left|z_{0}\right|^{N}$

+ terms in lower powers of $\left|z_{0}\right|$.

We have previously seen that if $\left|z_{0}\right|$ is sufficiently large we have relations of the forms

$$
\begin{aligned}
\mid\left[\text { v.a. } f_{M \prime \prime}(z), V_{1} V_{2}\right] \mid & \leqq \alpha_{1}\left|z_{0}\right|^{N-1}, \\
\mid\left[\text { v.a. } f_{0}(z), V_{3} V_{4}\right] \mid & \leqq \alpha_{2}\left|z_{0}\right|^{N-1}, \\
\mid\left[\text { v.a. }(h(z)+k(z)), V_{2} V_{3}\right] \mid & \leqq \alpha_{3}\left|z_{0}\right|^{N-1},
\end{aligned}
$$

where the $\alpha$ 's are positive constants. Also, if $\left|z_{0}\right|$ is large,

$$
\left|\mathfrak{\jmath}\left[g_{M}{ }^{\prime \prime}\left(z_{2}\right)-g_{0}\left(z_{3}\right)\right]\right| \leqq \alpha_{4}\left|z_{0}\right|^{N-1},
$$

where $\alpha_{4}$ is a positive constant.

It follows from the relations we have obtained that when $\left|z_{0}\right|$ is suffciently large we have

$$
Z_{f}(R)=\frac{\left|\mu_{M^{\prime \prime}}-\mu_{0}\right| \cdot\left|z_{0}\right|^{N}}{2 \pi}\left[1+O\left(1 /\left|z_{0}\right|\right)\right] .
$$

Equation (21) contains our fundamental result; from it Theorem 2 follows at once.

\subsection{Proof of Theorem 3}

We revert to the notation used in $\S 3$.

Suppose that the function $f_{\alpha}(z)$ has a critical ray lying in one of the sectors $S_{\alpha}{ }^{(0)}, \cdots, S_{\alpha}{ }^{(N-1)}$. We write 


$$
F_{\alpha}(z)=1+\sum_{m=0}^{M} \frac{f_{m}(z)}{f_{\alpha}(z)} \exp \left[g_{m}(z)-g_{\alpha}(z)+\left(\mu_{m}-\mu_{\alpha}\right) z^{N}\right],
$$

the prime on the summation sign indicating that the term for $m=\alpha$ is to be omitted. The zeros of $f(z)$ are the same as those of the function $f_{\alpha}(z) F_{\alpha}(z)$.

Our reasoning depends essentially on the following two theorems from the theory of integral functions*:

1. Let the integral function $F(z)$ be of finite order $\rho$. Let $z_{1}, z_{2}, z_{3}, \cdots$ denote the non-zero zeros of $F(z)$, and let $h$ be any real number greater than $\rho$. Then the series

$$
\sum_{i=1}^{\infty}\left|z_{i}\right|^{-h}
$$

converges.

2. Let the integral function $F(z)$ be of finite order $\rho$. Let $h$ be any positive number greater than $\rho$, and let $\epsilon$ be any positive number. About each of the nonzero zeros, $z_{i}$, of $F(z)$ as center describe a circle $\Gamma_{i}$ of radius $\left|z_{i}\right|^{-h}$. Then if the point $z$ is outside each of the circles $\Gamma_{i}$, and if $|z|$ is sufficiently large, we have the relation

$$
|F(z)|>\exp \left[-|z|^{\rho+\epsilon}\right] \text {. }
$$

It is to be noted that the first theorem insures that $z$ 's such as are referred to in the second theorem exist.

Obviously, the order of $f_{\alpha}(z)$ is not greater than $N_{\alpha}$. Let $h$ be a positive number greater than $N_{\alpha}$. Let $\epsilon$ be a positive number less than unity. By the second theorem cited above, if about each non-zero zero $z_{i}$ of $f_{\alpha}(z)$ as center we describe a circle $\Gamma_{i}$ of radius $\left|z_{i}\right|^{-h}$, and if we take $z$ outside of each of these circles, and such that $|z|$ is sufficiently large, we have

$$
\left|f_{\alpha}(z)\right|>\exp \left[-|z|^{N_{\alpha}+\epsilon}\right] \text {. }
$$

It follows readily from considerations similar to those used in the proof of Theorem 1 that if $z$ is in the sufficiently distant part of a certain sector $S$ that encloses the critical ray under consideration, we have a relation of the form

$$
\left|f_{\alpha}(z)\right| \cdot\left|F_{\alpha}(z)-1\right| \leqq \exp \left[-B|z|^{N}\right],
$$

where $B$ is a positive constant.

Let $k$ be any positive number less than unity. It is clear, from the foregoing, that we can find a positive number $K$ such that if $z$ is in the sector $S$, is outside of each of the circles $\Gamma_{i}$, and is such that $|z| \geqq K$, we have

$$
\left|F_{\alpha}(z)-1\right| \leqq k<1 \text {. }
$$

\footnotetext{
* Bieberbach, Lehrbuch der Funktionentheorie, vol. II, p. 243 and p. 268.
} 
We denote the set of all such points $z$ by the symbol $\Omega$.

Now consider any simple closed regular curve $\Gamma$ that is composed entirely of points of the set $\Omega$, and which does not pass through any zero of $f(z)$ or of $f_{\alpha}(z)$. The number, $Z_{f}(\Gamma)$, of zeros of $f(z)$ within $\Gamma$ is given by the formula

$$
Z_{f}(\Gamma)=\frac{1}{2 \pi}\left[\text { v.a. } f_{\alpha}(z), \Gamma\right]+\frac{1}{2 \pi}\left[\text { v.a. } F_{\alpha}(z), \Gamma\right] .
$$

The first term in the right-hand member of (23) is the number of zeros of $f_{\alpha}(z)$ within $\Gamma$. By (22), we have

$$
-\frac{1}{2}<\frac{1}{2 \pi}\left[\text { v.a. } F_{\alpha}(z), \Gamma\right]<\frac{1}{2} .
$$

Hence the number of zeros of $f(z)$ within $\Gamma$ is equal to the number of zeros of $f_{\alpha}(z)$ in the same region. This is our fundamental result.

Consider a rectangle bounded (1) by segments of length $r$ of the sides of the half-strip corresponding to the critical ray under consideration; (2) by the end of the half-strip; (3) by a segment congruent to the end. Let $V_{1}, V_{2}$, $V_{3}, V_{4}$ denote the vertices of the rectangle in counter-clockwise order, the segment $V_{4} V_{1}$ being the end of the half-strip. We consider three other rectangles, $V_{1} V_{2}^{\left({ }^{(0)}\right.} V_{3}^{\left({ }^{(0)}\right.} V_{4}, V_{1} V_{2}^{(1)} V_{3}^{(1)} V_{4}$, and $V_{1} V_{2}^{(2)} V_{3}^{(2)} V_{4}$. The segments $V_{2}^{(0)} V_{3}^{(0)}, V_{2}^{(1)} V_{3}^{(1)}, V_{2} V_{3}, V_{2}^{(2)} V_{3}^{(2)}$ are assumed to be crossed in that order as we proceed outward along the half-strip. The segments $V_{2}^{\left({ }^{(0)}\right.} V_{3}{ }^{(0)}, V_{2}^{(1)} V_{3}^{(1)}$, $V_{2}^{(2)} V_{3}^{(2)}, V_{2}^{(0)} V_{2}^{(2)}, V_{3}^{(0)} V_{3}^{(2)}$ are assumed to consist entirely of points of the set $\Omega{ }^{*}$ Furthermore, the boundaries of the rectangles $V_{2}^{(0)} V_{2}^{(1)} V_{3}^{(1)} V_{3}^{(0)}$, $V_{2}{ }^{(0)} V_{2}^{(2)} V_{3}^{(2)} V_{3}^{(0)}$ are assumed not to pass through any zeros of $f(z)$. Let the symbols $R, R_{0}, R_{1}, R_{2}$, respectively, denote the rectangles in the order in which they have been named. Let $R_{1}^{\prime}, R_{2}^{\prime}$ denote the rectangles $V_{2}^{\left({ }^{(0)}\right.} V_{2}^{(1)} V_{3}^{(1)} V_{3}^{(0)}, V_{2}^{(0)} V_{2}^{(2)} V_{3}^{(2)} V_{3}^{(0)}$, respectively.

Let $Z_{f}(R)$ denote the number of zeros of $f(z)$ within $R$. Similar symbols will be used in similar senses without further explanation.

Now obviously,

$$
\frac{Z_{f}\left(R_{1}^{\prime}\right)}{Z_{f_{\alpha}}\left(R_{0}\right)+Z_{f_{\alpha}}\left(R_{2}^{\prime}\right)} \leqq \frac{Z_{f}(R)}{Z_{f_{\alpha}}(R)} \leqq \frac{Z_{f}\left(R_{2}^{\prime}\right)}{Z_{f_{\alpha}}\left(R_{0}\right)+Z_{f_{\alpha}}\left(R_{1}^{\prime}\right)}+\frac{Z_{f}\left(R_{0}\right)}{Z_{f_{\alpha}}\left(R_{0}\right)+Z_{f_{\alpha}}\left(R_{1}^{\prime}\right)} .
$$

It has been shown that

$$
Z_{f}\left(R_{j}^{\prime}\right)=Z_{f_{\alpha}}\left(R_{j}^{\prime}\right), \quad j=1,2 .
$$

* It is a simple consequence of the first theorem cited above, and of our other results, that this condition can be satisfied. 
By the hypothesis for the induction, we have, for $j=1,2$,

$$
Z_{f_{\alpha}}\left(R_{j}^{\prime}\right)=\frac{a r_{j}^{n}}{2 \pi}\left[1+O\left(1 / r_{j}\right)\right]-\frac{a r_{0}^{n}}{2 \pi}\left[1+O\left(1 / r_{0}\right)\right]
$$

where $a$ is a positive constant, $n$ is a positive integer not greater than $N_{\alpha}$, and $r_{0}, r_{1}, r_{2}$ are the lengths of the segments $V_{1} V_{2}{ }^{(0)}, V_{1} V_{2}{ }^{(1)}, V_{1} V_{2}{ }^{(2)}$, respectively.

We hold $r_{0}$ fixed. By the first theorem cited above, if we make the segment $V_{1} V_{2}$ sufficiently long, we can make $r_{2}-r_{1}$ arbitrarily small without violating any of our previous stipulations. Now an easy and obvious calculation gives us the result stated in Theorem 3 .

BeLI TELEPHONE LABORATORIES, NEW YoRK, N. Y. 